\title{
Strategies to improve energy and power density of Li-ion batteries by virtual electrode design
}

\author{
$\underline{\text { Simon Hein }}{ }^{\mathrm{a}, \mathrm{b}}$, Timo Danner ${ }^{\mathrm{a}, \mathrm{b}}$, Shiying $\mathrm{Yu}^{\mathrm{c}}$, Daniel Westhoff ${ }^{\mathrm{d}}$, Benedikt Prifling ${ }^{\mathrm{d}}$, Sophie Sachse ${ }^{\mathrm{e}}$, \\ Wolfgang Haselrieder ${ }^{\mathrm{e}}$, Arno Kwade ${ }^{\mathrm{e}}$, Volker Schmidt ${ }^{\mathrm{d}}$, Arnulf Latz ${ }^{\mathrm{a}, \mathrm{b}, \mathrm{c}}$ \\ ${ }^{a}$ Helmholtz Institute Ulm for Electrochemical Energy Storage (HIU), Ulm, Germany \\ ${ }^{b}$ German Aerospace Center (DLR), Institute of Engineering Thermodynamics, Stuttgart, Germany \\ ${ }^{c}$ Ulm University, Institute of Electrochemistry, Ulm, Germany \\ ${ }^{d}$ Ulm University, Institute of Stochastics, Ulm, Germany \\ ${ }^{e}$ TU Braunschweig, Institut für Partikeltechnik, Braunschweig, Germany
}

\section{E-mail:simon.hein@dlr.de}

Technologically, a significant increase in energy density is a declared development goal for lithium-ion batteries for applications in electric vehicles. Developments are currently driven by the automotive industry and in order to reach their requirements for next-generation electric vehicles regarding safety, life-time, energy density, and fast charging further developments are inevitable. Additionally, a reduction of material and production costs is needed to improve the price competitiveness.

An attractive strategy to decrease the share of inactive materials is to increase the mass loading of the electrodes [1], [2]. This concept provides a high theoretical capacity and energy density. However, it also shifts the transport limitations of shuttling lithium ions in the electrolyte to lower C-rates and reduces the rate capability and practical capacity of the cell [2], [3]. In order to enable a fast charging of the batteries structuring techniques are investigated. A promising concept is the laser perforation of the electrodes which creates a hierarchical pore network with macroscopic transport pathways between anode and cathode. In this contribution we will present a study of an improved electrode design concept using laser perforation with the goal to optimize energy and power density of Li-lon battery electrodes and cells. As a first step, negative and positive electrodes consisting of synthetic graphite and nickel-rich NCM622, respectively, were manufactured in a continuous process.- After coating and drying, the electrodes were calendered and finally perforated with a dot-shaped pattern using a focused laser beam. In a next step reconstructions of the electrodes were created with the help of synchrotron tomography and a 3D stochastic microstructure generator [4], [5]. Furthermore, stochastic structure models were developed on the basis of the tomography data, which make it possible to investigate different structural scenarios. The resulting microstructures are then input to electrochemical simulations within our software BEST [6] and good qualitative agreement between the simulations and experimental data can be reported.

Our simulations confirm the beneficial effects which are found in the experiments. The impact of several design parameters including hole diameter, distance, and shape were investigated in order to find optimal conditions. Our study shows that this simulation-based approach is a powerful and efficient tool for the analysis and design of structured electrodes for Li-ion batteries.

\section{Acknowledgement}

This work has been funded by the 'Bundesministerium für Bildung und Forschung' within the project HighEnergy under the reference numbers 03XP0073D.

References:

[1] B. Delattre, R. Amin, J. Sander, J. De Coninck, A. P. Tomsia, and Y. Chiang, "Impact of Pore Tortuosity on Electrode Kinetics in Lithium Battery Electrodes: Study in Directionally FreezeCast LiNi 0.8 Co 0.15 Al 0.05 O 2 (NCA)," J. Electrochem. Soc., vol. 165, no. 2, pp. A388A395, Feb. 2018.

[2] M. Singh, J. Kaiser, and H. Hahn, "Thick Electrodes for High Energy Lithium Ion Batteries," J. Electrochem. Soc., vol. 162, no. 7, pp. A1196-A1201, 2015.

[3] T. Danner, M. Singh, S. Hein, J. Kaiser, H. Hahn, and A. Latz, "Thick electrodes for Li-ion batteries: A model based analysis," J. Power Sources, vol. 334, pp. 191-201, Dec. 2016.

[4] D. Westhoff et al., "Parametric stochastic 3D model for the microstructure of anodes in lithium-ion power cells," Comput. Mater. Sci., vol. 126, pp. 453-467, Jan. 2017. 
[5] S. Hein, J. Feinauer, D. Westhoff, I. Manke, V. Schmidt, and A. Latz, "Stochastic microstructure modeling and electrochemical simulation of lithium-ion cell anodes in 3D," $J$. Power Sources, vol. 336, pp. 161-171, Dec. 2016.

[6] A. Latz and J. Zausch, "Multiscale modeling of lithium ion batteries: thermal aspects," Beilstein J. Nanotechnol., vol. 6, pp. 987-1007, Apr. 2015. 